(c) 2010 IEEE. Personal use of this material is permitted. Permission from IEEE must be obtained for all other uses, in any current or future media, including reprinting/republishing this material for advertising or promotional purposes, creating new collective works, for resale or redistribution to servers or lists, or reuse of any copyrighted component of this work in other works. 


\section{Joint Source and Relay Optimization for Multiuser MIMO Relay Communication Systems}

\author{
Muhammad R. A. Khandaker \\ Dept. of Electrical and Computer Engineering \\ Curtin University of Technology \\ Bentley, WA-6102, Australia \\ Email: m.khandaker@postgrad.curtin.edu.au
}

\author{
Yue Rong \\ Dept. of Electrical and Computer Engineering \\ Curtin University of Technology \\ Bentley, WA-6102, Australia \\ Email: y.rong@curtin.edu.au
}

\begin{abstract}
In this paper, we design the source precoding matrices and the relay amplifying matrix of an amplify-andforward multiuser multiple-input multiple-output (MIMO) relay communication system. The minimum mean-squared error (MSE) is taken as the design criterion. We propose an alternating technique to efficiently solve the nonconvex source and relay optimization problem. It is shown that both the optimal source and relay matrices have a beamforming structure. Simulation results demonstrate that the proposed source and relay design algorithms perform much better than the existing techniques in terms of both MSE and bit-error-rate.
\end{abstract}

\section{INTRODUCTION}

Multiple-input multiple-output (MIMO) technique can provide spatial diversity and increase the spectral efficiency of wireless communication systems. Incorporating relays in a MIMO network can further increase the capacity, extend the coverage and improve the link reliability of the network. The capacity of a single-user MIMO relay channel has been studied in [1] and [2]. Several works studied the optimal relay amplifying matrix for a variety of objective functions when the amplify-and-forward (AF) relay strategy is used. In [3] and [4], the optimal relay amplifying matrix which maximizes the mutual information (MI) between source and destination was derived assuming that the source covariance matrix is an identity matrix. In [5] and [6], minimum mean-squared error (MMSE)-based approaches for MIMO relay systems have been studied. A unified framework was developed in [7] to jointly optimize the source precoding matrix and the relay amplifying matrix for a broad class of objective functions.

Recently, multiuser (MU) MIMO relay network has attracted much research interest. The achievable sum rate of a multiuser MIMO relay system has been studied in [8] and [9] using an AF relay scheme. In [10], both AF and decode-andforward (DF) relays have been considered in an MU-MIMO network without optimizing the power loading schemes at the relay and the source nodes. An adaptive relay power allocation algorithm has been considered in [11] in addition to self interference cancellation to achieve performance gain in an MU-MIMO relay network with DF relays. The optimal MSEbased joint filter design has been proposed for a multiuser AF MIMO relay system in [12]. But the system has significantly improved bit-error-rate (BER) performance only in the high signal-to-noise ratio (SNR) region. All these works with MUMIMO relay [8]-[12] assume that each user is equipped with a single antenna. Recently, the MU-MIMO relay system in [13] and [14] implemented multiple antennas at each user. In particular, [13] achieved much better BER performance at all the SNR regions, while the source and relay matrices were optimized in [14] to maximize the source-destination MI. However, the maximal MI-based algorithm is optimal only when the codewords are infinitely long. However, in practical communication systems, due to the delay constraint, codewords always have a finite length. Thus, the performance of the MI-based algorithm will degrade in practical systems.

In this paper, we study a multiuser MIMO relay communication system where each node is equipped with multiple antennas, and a linear receiver is used at the destination node. We develop the optimal structure of the source precoding matrices and relay amplifying matrix to jointly minimize the MSE of the signal waveform estimation at the destination node, which is closely related to the system raw BER. The optimization problem is nonconvex and therefore, a closedform solution is intractable. We use an alternating technique to optimize the system performance and show that the optimal source and relay matrices have a beamforming structure. Simulation results demonstrate that the proposed iterative source and relay optimizing algorithm performs much better than existing techniques in terms of both MSE and BER.

The rest of this paper is organized as follows. In Section II, the system model of an MU-MIMO relay network is introduced; the optimal structure of the source precoding matrices and relay amplifying matrix is developed in Section III; Section IV shows the simulation results which justify the significance of the proposed algorithm under various scenarios and finally, conclusions are drawn in Section V.

\section{SySTEM MOdEL}

We consider a two-hop multiuser MIMO relay communication system as illustrated in Fig. 1 where $N_{u}$ users transmit information to the same destination node with the aid of one relay node. The $i$ th user, $i=1, \cdots, N_{u}$, the relay and 
the destination nodes are equipped with $N_{s_{i}}, N_{r}$, and $N_{d}$ antennas, respectively. We denote $N_{b}=\sum_{i=1}^{N_{u}} N_{s_{i}}$ as the number of independent data streams from all the users to the relay. To efficiently exploit the system hardware, the relay node uses the same antennas to transmit and receive signals. For simplicity, the AF strategy is applied at the relay node to process and forward the received signals.

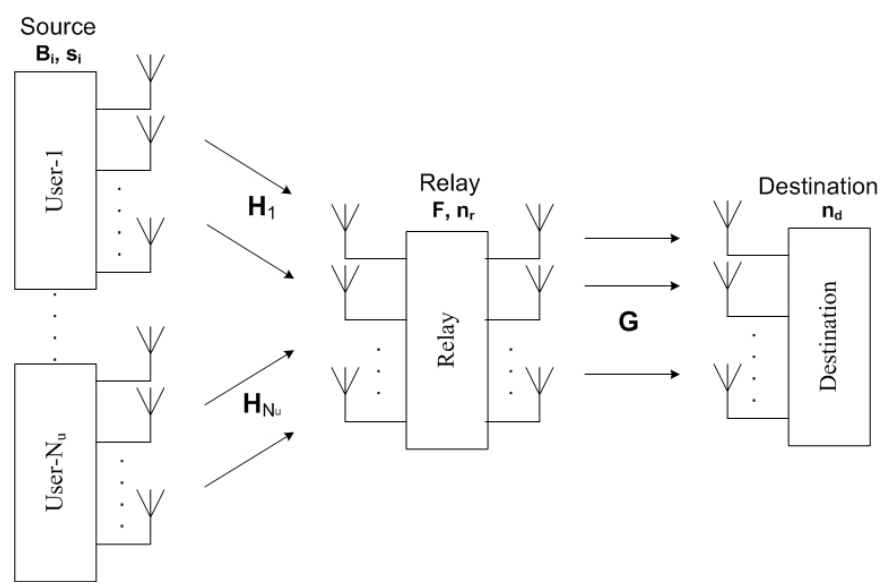

Fig. 1. Block diagram of an $N_{u}$-user AF MIMO relay communications system.

We make the common assumption that the relay node works in the half-duplex mode. Thus, the communication between the source and destination is completed in two time slots. In the first time slot, the $N_{s_{i}} \times 1$ modulated signal vector $\mathbf{s}_{i}$ is linearly precoded at the $i$ th user by the $N_{s_{i}} \times N_{s_{i}}$ source precoding matrix $\mathbf{B}_{i}, i=1, \cdots, N_{u}$. The precoded vector

$$
\mathbf{x}_{i}=\mathbf{B}_{i} \mathbf{s}_{i}
$$

is transmitted to the relay node from the $i$ th user. Thus the received signal at the relay node can be written as

$$
\mathbf{y}_{r}=\sum_{i=1}^{N_{u}} \mathbf{H}_{i} \mathbf{x}_{i}+\mathbf{n}_{r}
$$

where $\mathbf{H}_{i}$ is the $N_{r} \times N_{s_{i}}$ MIMO channel matrix between the $i$ th user and the relay, $\mathbf{y}_{r}$ and $\mathbf{n}_{r}$ are the received signal and the additive Gaussian noise vectors at the relay node, respectively.

In the second time slot, the users remain silent and the relay node multiplies (linearly precodes) the received signal vector $\mathbf{y}_{r}$ by an $N_{r} \times N_{r}$ relay amplifying matrix $\mathbf{F}$ and transmits the precoded signal vector

$$
\mathbf{x}_{r}=\mathbf{F} \mathbf{y}_{r}
$$

to the destination node. Hence the received signal vector at the destination node can be written as

$$
\mathbf{y}_{d}=\mathbf{G x}_{r}+\mathbf{n}_{d}
$$

where $\mathbf{G}$ is the $N_{d} \times N_{r}$ MIMO channel matrix between the relay and the destination nodes, $\mathbf{y}_{d}$ and $\mathbf{n}_{d}$ are the received signal and the additive Gaussian noise vectors at the destination node, respectively.
Substituting (1)-(3) into (4), we obtain

$$
\begin{aligned}
\mathbf{y}_{d} & =\mathbf{G F} \sum_{i=1}^{N_{u}} \mathbf{H}_{i} \mathbf{B}_{i} \mathbf{s}_{i}+\mathbf{G F} \mathbf{n}_{r}+\mathbf{n}_{d} \\
& =\left[\mathbf{G F H}_{1} \mathbf{B}_{1}, \cdots, \mathbf{G F H}_{N_{u}} \mathbf{B}_{N_{u}}\right]\left[\begin{array}{c}
\mathbf{s}_{1} \\
\vdots \\
\mathbf{s}_{N_{u}}
\end{array}\right]+\mathbf{G F n}_{r}+\mathbf{n}_{d} \\
& =\mathbf{H} \mathbf{s}+\mathbf{n}
\end{aligned}
$$

where $\mathbf{H} \triangleq\left[\mathbf{G F H}_{1} \mathbf{B}_{1}, \cdots, \mathbf{G F H}_{N_{u}} \mathbf{B}_{N_{u}}\right]$ is the equivalent MIMO channel matrix of the source-relay-destination link, $\mathbf{s} \triangleq$ $\left[\mathbf{s}_{1}^{T}, \cdots, \mathbf{s}_{N_{u}}^{T}\right]^{T}$ is the equivalent transmitted signal vector, and $\mathbf{n} \triangleq \mathbf{G F n}_{r}+\mathbf{n}_{d}$ is the equivalent noise vector. Here $(\cdot)^{T}$ indicates the transpose of a matrix.

We assume that the channel matrices $\mathbf{H}_{i}, i=1, \cdots, N_{u}$, and $\mathbf{G}$ are all quasi-static and known to the relay and the destination nodes. In practice, the channel state information (CSI) of $\mathbf{G}$ can be obtained at the destination node through standard training method. The relay node can have the CSI of $\mathbf{H}_{i}$ through channel training, and obtain the CSI of $\mathbf{G}$ by a feedback from the destination node. For wireless relays, the fading is often relatively slow whenever the mobility of the relays is relatively low, and for static relays, the channel state information can be almost constant. Thus, in this way, the necessary CSI can be obtained with a reasonably high precision. The relay node calculates the optimal source $\left(\mathbf{B}_{i}, i=1, \cdots, N_{u},\right)$ and relay $(\mathbf{F})$ matrices, and then forwards $\mathbf{B}_{i}$ to user $i$ and forwards $\mathbf{F}$ and $\mathbf{H}$ to the destination node. We also assume that all noises are independent and identically distributed (i.i.d.) complex circularly symmetric Gaussian noise with zero mean and unit variance.

For simplicity, a linear receiver is used at the destination node to retrieve the transmitted signals. Thus the estimated signal waveform is given by

$$
\hat{\mathbf{s}}=\mathbf{W}^{H} \mathbf{y}_{d}
$$

where $\mathbf{W}$ is an $N_{d} \times N_{b}$ weight matrix, and $(\cdot)^{H}$ denotes matrix (vector) Hermitian transpose.

\section{Optimal Source And Relay Design}

In this section we develop the optimal structure of the source precoding matrix $\mathbf{B}_{i}, i=1, \cdots, N_{u}$, and the relay amplifying matrix $\mathbf{F}$ to minimize the MSE of the signal waveform estimation, which is closely related to the system raw BER.

Using the linear receiver in (5), the MSE of the signal waveform estimation at the destination is given by

$$
\begin{aligned}
\mathrm{MSE}= & \operatorname{tr}\left\{E\left[(\hat{\mathbf{s}}-\mathbf{s})(\hat{\mathbf{s}}-\mathbf{s})^{H}\right]\right\} \\
= & \operatorname{tr}\left\{\left(\mathbf{W}^{H} \mathbf{H}-\mathbf{I}_{N_{b}}\right)\left(\mathbf{W}^{H} \mathbf{H}-\mathbf{I}_{N_{b}}\right)^{H}\right. \\
& \left.+\mathbf{W}^{H} \mathbf{C}_{n} \mathbf{W}\right\}
\end{aligned}
$$

where $\operatorname{tr}\{\cdot\}$ is the trace of a matrix, $E[\cdot]$ stands for the statistical expectation, and $\mathbf{I}_{n}$ is an $n \times n$ identity matrix. Here 
we assumed that $E\left[\mathbf{s s}^{H}\right]=\mathbf{I}_{N_{b}}$, and $\mathbf{C}_{n}$ is the equivalent noise covariance matrix given by

$$
\begin{aligned}
\mathbf{C}_{n} & =E\left[\mathbf{n n}^{H}\right] \\
& =E\left[\left(\mathbf{G F} \mathbf{n}_{r}+\mathbf{n}_{d}\right)\left(\mathbf{G} \mathbf{F} \mathbf{n}_{r}+\mathbf{n}_{d}\right)^{H}\right] \\
& =\mathbf{G F F} \mathbf{G}^{H}+\mathbf{I}_{N_{d}} .
\end{aligned}
$$

The weight matrix of the optimal linear receiver which minimizes MSE in (6) is essentially the Wiener filter given by [15]

$$
\mathbf{W}=\left(\mathbf{H} \mathbf{H}^{H}+\mathbf{C}_{n}\right)^{-1} \mathbf{H}
$$

where $(\cdot)^{-1}$ denotes matrix inversion. Substituting (7) back into (6), we obtain the minimal MSE as a function of $\mathbf{B}_{i}$, $i=1, \cdots, N_{u}$, and $\mathbf{F}$, given by

$$
\mathrm{MMSE}=\operatorname{tr}\left\{\mathbf{I}_{N_{b}}-\mathbf{H}^{H}\left(\mathbf{H H}^{H}+\mathbf{C}_{n}\right)^{-1} \mathbf{H}\right\} .
$$

Applying the matrix inversion lemma $(\mathbf{A}+\mathbf{B C D})^{-1}=$ $\mathbf{A}^{-1}-\mathbf{A}^{-1} \mathbf{B}\left(\mathbf{D A}{ }^{-1} \mathbf{B}+\mathbf{C}^{-1}\right)^{-1} \mathbf{D} \mathbf{A}^{-1}$, (8) can be written as

$$
\mathrm{MMSE}=\operatorname{tr}\left\{\left[\mathbf{I}_{N_{b}}+\mathbf{H}^{H} \mathbf{C}_{n}^{-1} \mathbf{H}\right]^{-1}\right\} .
$$

Considering (3), the transmission power consumed by the relay node can be expressed as

$$
\operatorname{tr}\left\{E\left[\mathbf{x}_{r} \mathbf{x}_{r}^{H}\right]\right\}=\operatorname{tr}\left\{\mathbf{F}\left(\sum_{i=1}^{N_{u}} \mathbf{H}_{i} \mathbf{B}_{i} \mathbf{B}_{i}^{H} \mathbf{H}_{i}^{H}+\mathbf{I}_{N_{r}}\right) \mathbf{F}^{H}\right\} .
$$

Thus the relay amplifying matrix and source precoding matrices optimization problem can be formulated as

$$
\begin{gathered}
\min _{\mathbf{B}_{1}, \cdots, \mathbf{B}_{N_{u}}, \mathbf{F}} \operatorname{tr}\left\{\left[\mathbf{I}_{N_{b}}+\mathbf{H}^{H} \mathbf{C}_{n}^{-1} \mathbf{H}\right]^{-1}\right\} \\
\text { s.t. } \quad \operatorname{tr}\left\{\mathbf{F}\left(\sum_{i=1}^{N_{u}} \mathbf{H}_{i} \mathbf{B}_{i} \mathbf{B}_{i}^{H} \mathbf{H}_{i}^{H}+\mathbf{I}_{N_{r}}\right) \mathbf{F}^{H}\right\} \leq P_{r} \\
\operatorname{tr}\left\{\mathbf{B}_{i} \mathbf{B}_{i}^{H}\right\} \leq P_{s, i}, \quad i=1, \cdots, N_{u}
\end{gathered}
$$

where (10) and (11) are the constraints for the transmission power at the relay and $i$ th user, respectively, and $P_{r}>0$, $P_{s, i}>0$ are the power budget available at the relay and the $i$ th source node, respectively. The optimization problem (9)(11) is nonconvex and a closed-form solution to the problem is intractable. In this paper, we develop an iterative (alternating) algorithm to optimize the source and the relay matrices.

\section{A. Relay-only Optimization}

For given source matrices, $\mathbf{B}_{i}, i=1, \cdots, N_{u}$, satisfying (11), we optimize the relay matrix $\mathbf{F}$ by solving the following problem

$$
\begin{aligned}
& \min _{\mathbf{F}} \operatorname{tr}\left\{\left[\mathbf{I}_{N_{b}}+\mathbf{H}^{H} \mathbf{C}_{n}^{-1} \mathbf{H}\right]^{-1}\right\} \\
& \text { s.t. } \operatorname{tr}\left\{\mathbf{F}\left(\sum_{i=1}^{N_{u}} \mathbf{H}_{i} \mathbf{B}_{i} \mathbf{B}_{i}^{H} \mathbf{H}_{i}^{H}+\mathbf{I}_{N_{r}}\right) \mathbf{F}^{H}\right\} \leq P_{r} .
\end{aligned}
$$

Let us now define the following singular value decompositions (SVDs)

$$
\begin{aligned}
{\left[\mathbf{H}_{1} \mathbf{B}_{1}, \mathbf{H}_{2} \mathbf{B}_{2}, \cdots, \mathbf{H}_{N_{u}} \mathbf{B}_{N_{u}}\right] } & =\mathbf{U}_{s} \boldsymbol{\Lambda}_{s} \mathbf{V}_{s}^{H} \\
\mathbf{G} & =\mathbf{U}_{r} \boldsymbol{\Lambda}_{r} \mathbf{V}_{r}^{H}
\end{aligned}
$$

where the dimensions of $\mathbf{U}_{s}, \boldsymbol{\Lambda}_{s}, \mathbf{V}_{s}$ are $N_{r} \times N_{r}, N_{r} \times N_{b}$, $N_{b} \times N_{b}$, respectively, and the dimensions of $\mathbf{U}_{r}, \boldsymbol{\Lambda}_{r}, \mathbf{V}_{r}$ are given as $N_{d} \times N_{d}, N_{d} \times N_{r}, N_{r} \times N_{r}$, respectively. We assume that the main diagonal elements of $\boldsymbol{\Lambda}_{s}$ and $\boldsymbol{\Lambda}_{r}$ are arranged in decreasing order. Based on the theorem in [7], the optimal structure of $\mathbf{F}$ is given by

$$
\mathbf{F}=\mathbf{V}_{r, 1} \boldsymbol{\Lambda}_{f} \mathbf{U}_{s, 1}^{H}
$$

where $\boldsymbol{\Lambda}_{f}$ is an $N_{b} \times N_{b}$ diagonal matrix, $\mathbf{V}_{r, 1}$ and $\mathbf{U}_{s, 1}$ contain the leftmost $N_{b}$ columns from $\mathbf{V}_{r}$ and $\mathbf{U}_{s}$, respectively.

\section{B. Joint Source and Relay Optimization}

Once the optimal $\mathbf{F}$ is calculated following the unified framework in [7], the optimal $\mathbf{B}_{i}, i=1, \cdots, N_{u}$, should be found to solve the problem (9)-(11). The objective function (9) can be rewritten as

$$
\begin{aligned}
\text { MMSE }= & \operatorname{tr}\left\{\left[\mathbf{I}_{N_{d}}+\mathbf{C}_{n}^{-\frac{1}{2}} \mathbf{G F} \sum_{i=1}^{N_{u}} \mathbf{H}_{i} \mathbf{B}_{i} \mathbf{B}_{i}^{H} \mathbf{H}_{i}^{H} \mathbf{F}^{H}\right.\right. \\
& \left.\left.\times \mathbf{G}^{H} \mathbf{C}_{n}^{-\frac{1}{2}}\right]^{-1}\right\}+N_{b}-N_{d} \\
= & \operatorname{tr}\left\{\left[\mathbf{I}_{N_{d}}+\sum_{i=1}^{N_{u}} \tilde{\mathbf{H}}_{i} \mathbf{Q}_{i} \tilde{\mathbf{H}}_{i}^{H}\right]^{-1}\right\}+N_{b}-N_{d}
\end{aligned}
$$

where $\tilde{\mathbf{H}}_{i} \triangleq \mathbf{C}_{n}^{-\frac{1}{2}} \mathbf{G} \mathbf{F} \mathbf{H}_{i}$ and $\mathbf{Q}_{i}=\mathbf{B}_{i} \mathbf{B}_{i}^{H}$ is the source covariance matrix of the $i$ th user. The source covariance matrices can be optimized by solving the following problem

$$
\begin{aligned}
\min _{\mathbf{Q}_{1}, \cdots, \mathbf{Q}_{N_{u}}} & \operatorname{tr}\left\{\left[\mathbf{I}_{N_{d}}+\sum_{i=1}^{N_{u}} \tilde{\mathbf{H}}_{i} \mathbf{Q}_{i} \tilde{\mathbf{H}}_{i}^{H}\right]^{-1}\right\}+N_{b}-N_{d}(15) \\
\text { s.t. } & \operatorname{tr}\left\{\sum_{i=1}^{N_{u}} \mathbf{Q}_{i} \mathbf{\Psi}_{i}\right\} \leq \bar{P}_{r} \\
& \operatorname{tr}\left\{\mathbf{Q}_{i}\right\} \leq P_{s, i}, \quad i=1, \cdots, N_{u} \\
& \mathbf{Q}_{i} \geq 0, \quad i=1, \cdots, N_{u}
\end{aligned}
$$

where $\boldsymbol{\Psi}_{i} \triangleq \mathbf{H}_{i}^{H} \mathbf{F}^{H} \mathbf{F} \mathbf{H}_{i}, \bar{P}_{r} \triangleq P_{r}-\operatorname{tr}\left\{\mathbf{F} \mathbf{F}^{H}\right\}$.

Let us now introduce a matrix $\mathbf{X}$ that satisfies

$$
\left[\mathbf{I}_{N_{d}}+\sum_{i=1}^{N_{u}} \tilde{\mathbf{H}}_{i} \mathbf{Q}_{i} \tilde{\mathbf{H}}_{i}^{H}\right]^{-1} \leq \mathbf{X}
$$

where for two matrices $\mathbf{A}$ and $\mathbf{B}, \mathbf{B} \geq \mathbf{A}$ means that $\mathbf{B}-\mathbf{A}$ is a positive semi-definite matrix (i.e., $\mathbf{B}-\mathbf{A} \geq 0$ ). By using (19) and the Schur complement [16], the problem (15)-(17) can be equivalently converted to the following semi-definite 
programming (SDP) problem

$$
\begin{aligned}
& \min _{\mathbf{Q}_{1}, \cdots, \mathbf{Q}_{N_{u}}, \mathbf{X}} \operatorname{tr}\{\mathbf{X}\} \\
& \text { s.t. }\left[\begin{array}{ll}
\mathbf{X} & \mathbf{I}_{N_{d}} \\
\mathbf{I}_{N_{d}} & \mathbf{I}_{N_{d}}+\sum_{i=1}^{N_{u}} \tilde{\mathbf{H}}_{i} \mathbf{Q}_{i} \tilde{\mathbf{H}}_{i}^{H}
\end{array}\right] \geq 0 \\
& \operatorname{tr}\left\{\sum_{i=1}^{N_{u}} \mathbf{Q}_{i} \boldsymbol{\Psi}_{i}\right\} \leq \bar{P}_{r} \\
& \operatorname{tr}\left\{\mathbf{Q}_{i}\right\} \leq P_{s, i}, \quad i=1, \cdots, N_{u} \\
& \mathbf{Q}_{i} \geq 0, \quad i=1, \cdots, N_{u} \text {. }
\end{aligned}
$$

Several software packages are available to solve SDP problems like (20)-(24). We used CVX MATLAB toolbox for disciplined convex programming [17] to optimize $\mathbf{Q}_{i}, i=$ $1, \cdots, N_{u}$.

Now the original source and relay matrices optimization problem (9)-(11) can be solved by an iterative technique as shown in Table I. Here $\|\cdot\|_{1}$ denotes the matrix maximum absolute column sum norm, $\varepsilon$ is a small positive number close to zero and the superscript $(n)$ denotes the number of iterations.

\section{TABLE I}

PROCEDURE OF SOLVING THE PROBLEM (9)-(11) BY THE PROPOSED ITERATIVE ALGORITHM

1) Initialize the algorithm with randomly generated $\mathbf{Q}_{i}^{(0)}, i=$ $1, \cdots, N_{u}$, meeting power constraint (17); Set $n=0$.

2) Solve the subproblem (12)-(13) using given $\mathbf{Q}_{i}^{(n)}, i=1, \cdots, N_{u}$, to obtain $\mathbf{F}^{(n)}$ as in (14).

3) Solve the subproblem (20)-(24) using known $\mathbf{F}^{(n)}$ to obtain $\mathbf{Q}_{i}^{(n+1)}$, $i=1, \cdots, N_{u}$

4) If $\max _{i}\left\|\mathbf{Q}_{i}^{(n+1)}-\mathbf{Q}_{i}^{(n)}\right\|_{1} \leq \varepsilon$, then end Otherwise, let $n:=n+1$ and go to step 2 .

The conditional updates of $\mathbf{F}$ and $\mathbf{Q}_{i}$ may either decrease or maintain but cannot increase the objective function (9). Monotonic convergence of $\mathbf{F}$ and $\mathbf{Q}_{i}$ follows directly from this observation. The numerical solution to the problem (20)(24) does not provide sufficient insight to the structure of the optimal $\mathbf{Q}_{i}$. By solving the problem (15)-(17) applying the Lagrange multiplier method, we obtain the following theorem for the structure of the optimal $\mathbf{Q}_{i}$.

THEOREM 1: The optimal source covariance matrix $\mathbf{Q}_{i}$ for $i$ th user as the solution to the problem (15)-(17) has the following general beamforming structure

$$
\begin{array}{r}
\mathbf{Q}_{i}=\mathbf{V}_{h_{i}} \boldsymbol{\Lambda}_{h_{i}, 1}^{-1} \mathbf{U}_{h_{i}, 1}^{H}\left(\mathbf{V}_{i} \mathbf{J}_{i} \mathbf{V}_{i}^{H}-\mathbf{D}_{i}\right)^{+} \mathbf{U}_{h_{i}, 1} \boldsymbol{\Lambda}_{h_{i}, 1}^{-1} \mathbf{V}_{h_{i}}^{H} \\
i=1, \cdots, N_{u}
\end{array}
$$

where $\mathbf{D}_{i} \triangleq \mathbf{I}_{N_{d}}+\sum_{j=1, j \neq i}^{N_{u}} \tilde{\mathbf{H}}_{j} \mathbf{Q}_{j} \tilde{\mathbf{H}}_{j}^{H},(\cdot)^{+}$stands for the projection to the set of positive semi-definite matrices, $\tilde{\mathbf{H}}_{i}=\left[\begin{array}{ll}\mathbf{U}_{h_{i}, 1} & \mathbf{U}_{h_{i}, 2}\end{array}\right]\left[\begin{array}{ll}\boldsymbol{\Lambda}_{h_{i}, 1} & \mathbf{0}\end{array}\right]^{T} \mathbf{V}_{h_{i}}^{H}$ and $\mathbf{K}_{i}^{-1} \tilde{\mathbf{H}}_{i}^{H}=$ $\mathbf{U}_{i}\left[\begin{array}{ll}\boldsymbol{\Sigma}_{i} & \mathbf{0}\end{array}\right] \mathbf{V}_{i}^{H}$ are the SVDs of $\tilde{\mathbf{H}}_{i}$ and $\mathbf{K}_{i}^{-1} \tilde{\mathbf{H}}_{i}^{H}$, respectively, and $\mathbf{J}_{i} \triangleq \operatorname{Bdiag}\left[\boldsymbol{\Sigma}_{i}, \boldsymbol{\Delta}_{i, 2}\right]$. Here $\mathbf{K}_{i} \mathbf{K}_{i}^{H}=\lambda_{1} \boldsymbol{\Psi}_{i}+$ $\lambda_{2} \mathbf{I}_{N_{s_{i}}}, \lambda_{1} \geq 0, \lambda_{2} \geq 0$ are the Lagrange multipliers, and Bdiag[.] stands for a block diagonal matrix.

PRoOF: See Appendix A.
The unknown Lagrange multipliers $\lambda_{1}$ and $\lambda_{2}$ in (25) can be found by solving the dual optimization problem associated with the problem (26)-(29) in Appendix A.

Once we get the optimal source covariance matrix, the optimal source precoding matrix for $i$ th user is calculated as

$$
\mathbf{B}_{i}=\mathbf{U}_{q, i} \boldsymbol{\Lambda}_{q, i}^{\frac{1}{2}}, \quad i=1, \cdots, N_{u}
$$

with the following eigenvalue decomposition (EVD) of the optimal source covariance matrix

$$
\begin{gathered}
\mathbf{Q}_{i}=\mathbf{U}_{q, i} \boldsymbol{\Lambda}_{q, i} \mathbf{U}_{q, i}^{H}, \quad i=1, \cdots, N_{u} . \\
\text { IV. Simulations }
\end{gathered}
$$

In this section, we study the performance of the proposed source precoding matrices and relay amplifying matrix through numerical simulations. For simplicity, we consider here a system with two users. The extension to $N_{u}\left(N_{u}>2\right)$ users is straight-forward. The two users, relay and destination nodes are all equipped with multiple antennas. We simulate a flat Rayleigh fading environment where the channel matrices have entries with zero mean and variances $\sigma_{g}^{2} / N_{r}, \sigma_{h, i}^{2} / N_{s_{i}}$, $i=1,2$, for $\mathbf{G}, \mathbf{H}_{i}, i=1,2$, respectively. We define

$\mathrm{SNR}_{\mathrm{r}-\mathrm{d}} \triangleq \frac{\sigma_{g}^{2} P_{r} N_{d}}{N_{r}}, \quad \mathrm{SNR}_{\mathrm{s}_{\mathrm{i}}-\mathrm{r}} \triangleq \frac{\sigma_{h, i}^{2} P_{s, i} N_{r}}{N_{s_{i}}}, \quad i=1,2$ as the SNR of the relay-destination and user- $i$-relay links, $i=$ 1,2 , respectively. For simplicity, we assume $N_{s_{1}}=N_{s_{2}}=N_{s}$ and $\mathrm{SNR}_{\mathrm{S}_{1}-\mathrm{r}}=\mathrm{SNR}_{\mathrm{S}_{2}-\mathrm{r}}=\mathrm{SNR}_{\mathrm{S}-\mathrm{r}}$ in all simulations. All simulation results are averaged over 200 independent channel realizations.

We compare the performance of the proposed joint optimal algorithm (Optimal B \& F) with the relay-only optimal algorithm (Optimal F), the naive amplify-and-forward (NAF) algorithm, and the pseudo match-and-forward (PMF) algorithm. For the joint optimal algorithm, the procedure in Table $\mathrm{I}$ is carried out to obtain the optimal relay and source matrices. To initialize the algorithm in Table I, we randomly generate 10 independent $\mathbf{Q}_{1}$ and $\mathbf{Q}_{2}$ meeting the power constraint (17) and choose the one that yields the minimum MSE. For the relay-only optimal algorithm, we used the optimal $\mathbf{F}$ from the first iteration and the initial $\mathbf{B}_{i}$ of the joint optimal algorithm. For the NAF scheme, we used the same $\mathbf{B}_{i}$ as for the relayonly optimal algorithm and

$$
\mathbf{F}=\sqrt{\frac{P_{r}}{\operatorname{tr}\left\{\overline{\mathbf{H}} \overline{\mathbf{H}}^{H}+\mathbf{I}_{N_{r}}\right\}}} \mathbf{I}_{N_{r}}
$$

with $\overline{\mathbf{H}}=\left[\mathbf{H}_{1} \mathbf{B}_{1}, \cdots, \mathbf{H}_{N_{u}} \mathbf{B}_{N_{u}}\right]$. For the PMF algorithm, the same $\mathbf{B}_{i}$ as for the relay-only optimal algorithm is taken and

$$
\mathbf{F}=\sqrt{\frac{P_{r}}{\operatorname{tr}\left\{(\overline{\mathbf{H}} \mathbf{G})^{H}\left(\overline{\mathbf{H}} \overline{\mathbf{H}}^{H}+\mathbf{I}_{N_{r}}\right) \overline{\mathbf{H}} \mathbf{G}\right\}}}(\overline{\mathbf{H}} \mathbf{G})^{H} .
$$

Fig. 2 shows the MSE performance of all algorithms versus $\mathrm{SNR}_{\mathrm{s}-\mathrm{r}}$ with $N_{s}=3, N_{r}=N_{d}=6$, and $\mathrm{SNR}_{\mathrm{r}-\mathrm{d}}=20 \mathrm{~dB}$, whereas Fig. 3 shows the MSE performance versus $\mathrm{SNR}_{\mathrm{r}-\mathrm{d}}$ with $N_{s}=3, N_{r}=N_{d}=6$, and $\mathrm{SNR}_{\mathrm{s}-\mathrm{r}}=20 \mathrm{~dB}$. 


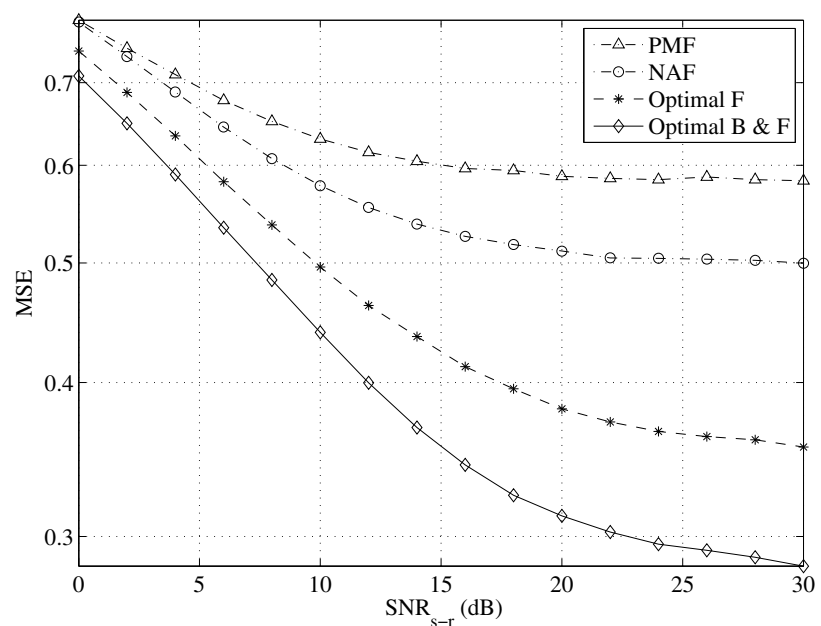

Fig. 2. MSE versus $\mathrm{SNR}_{\mathrm{s}-\mathrm{r}} . N_{s}=3, N_{r}=N_{d}=6, \mathrm{SNR}_{\mathrm{r}-\mathrm{d}}=20 \mathrm{~dB}$.

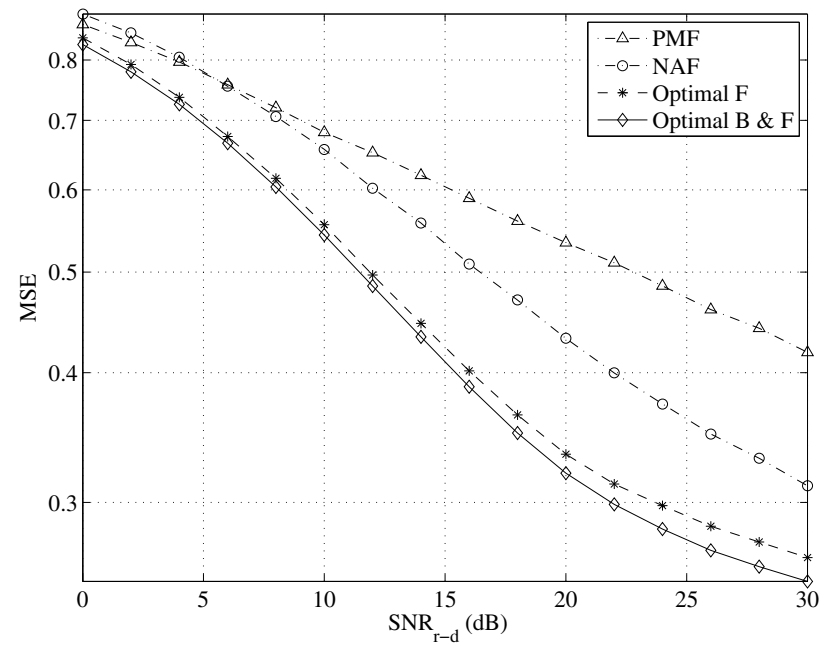

Fig. 3. MSE versus $\mathrm{SNR}_{\mathrm{r}-\mathrm{d}} \cdot N_{s}=3, N_{r}=N_{d}=6, \mathrm{SNR}_{\mathrm{s}-\mathrm{r}}=20 \mathrm{~dB}$

Our results clearly demonstrate the better performance of the proposed iterative joint source and relay optimization technique. It can be seen that the proposed optimal algorithm consistently yields the lowest MSE over the whole SNR range. The NAF and PMF algorithms have much higher MSE compared with the other algorithms even at very high SNR. We also find that the proposed joint optimal algorithm yields significant improvement over the relay-only optimal scheme.

In the next example, we compare the performance of different algorithms in terms of BER. QPSK signal constellations are used to modulate the transmitting signals. We transmit $N_{s} \times 10^{3}$ randomly generated bits from each user in each channel realization. Fig. 4 shows the BER performance of three algorithms versus $\mathrm{SNR}_{\mathrm{s}-\mathrm{r}}$ for $\mathrm{SNR}_{\mathrm{r}-\mathrm{d}}=20 \mathrm{~dB}$ and $N_{s}=2, N_{r}=N_{d}=8$. Note that in contrast to other three schemes, the PMF algorithm requires $N_{b}=N_{d}$, and thus, its performance cannot be included in Fig. 4. It can be seen from

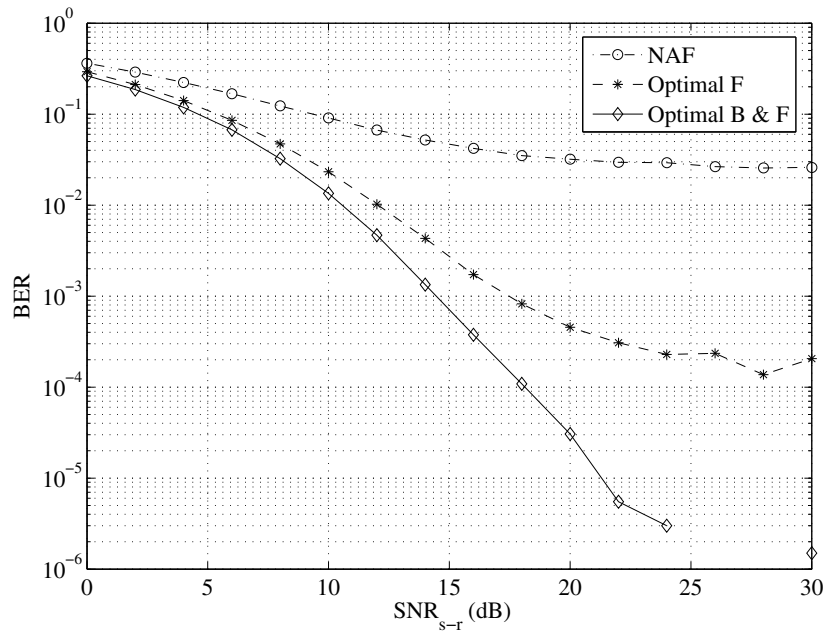

Fig. 4. BER versus $\mathrm{SNR}_{\mathrm{s}-\mathrm{r}} . N_{s}=2, N_{r}=N_{d}=8, \mathrm{SNR}_{\mathrm{r}-\mathrm{d}}=20 \mathrm{~dB}$.

Fig. 4 that the proposed joint source and relay optimization technique obtains the lowest BER compared with the other approaches.

\section{CONCLUSIONS}

We derived the optimal structure of the source precoding matrices and the relay amplifying matrix in a multiuser MIMO relay network to jointly minimize the MSE of the signal waveform estimation. Since the optimization problem is nonconvex, there is no closed-form solution. We developed an iterative technique to optimize the source and relay matrices. Simulation results demonstrate that the joint optimal source and relay algorithm outperforms the existing techniques in terms of MSE and BER. Future works may include considering the direct link between the source and the destination and using multiple relays in an MU-MIMO network.

\section{APPENDIX A}

PRoOF OF THEOREM 1

To determine the structure of the optimal source covariance matrix $\mathbf{Q}_{i}$ for the $i$ th user, we rewrite the problem (15)-(17) with given $\mathbf{Q}_{j}, j=1, \cdots, N_{u}, j \neq i$ as

$$
\begin{array}{ll}
\min _{\mathbf{Q}_{i}} & \operatorname{tr}\left\{\left[\mathbf{D}_{i}+\tilde{\mathbf{H}}_{i} \mathbf{Q}_{i} \tilde{\mathbf{H}}_{i}^{H}\right]^{-1}\right\} \\
\text { s.t. } & \operatorname{tr}\left\{\mathbf{Q}_{i} \mathbf{\Psi}_{i}\right\} \leq \tilde{P}_{r} \\
& \operatorname{tr}\left\{\mathbf{Q}_{i}\right\} \leq P_{s, i} \\
& \mathbf{Q}_{i} \geq 0
\end{array}
$$

where $\tilde{P}_{r} \triangleq \bar{P}_{r}-\operatorname{tr}\left\{\sum_{j=1, j \neq i}^{N_{u}} \mathbf{Q}_{j} \mathbf{\Psi}_{j}\right\}$. The Lagrangian function associated with the problem (26)-(28) is given by

$$
\begin{aligned}
\mathcal{L}= & \operatorname{tr}\left\{\left[\mathbf{D}_{i}+\tilde{\mathbf{H}}_{i} \mathbf{Q}_{i} \tilde{\mathbf{H}}_{i}^{H}\right]^{-1}\right\}+\lambda_{1}\left(\operatorname{tr}\left\{\mathbf{Q}_{i} \mathbf{\Psi}_{i}\right\}-\tilde{P}_{r}\right) \\
& +\lambda_{2}\left(\operatorname{tr}\left\{\mathbf{Q}_{i}\right\}-P_{s, i}\right)
\end{aligned}
$$


where $\lambda_{1} \geq 0$ and $\lambda_{2} \geq 0$ are the Lagrange multipliers. Making the derivative of $\mathcal{L}$ with respect to $\mathbf{Q}_{i}$ be zero, we obtain

$\frac{\partial \mathcal{L}}{\partial \mathbf{Q}_{i}}=-\tilde{\mathbf{H}}_{i}^{H}\left(\mathbf{D}_{i}+\tilde{\mathbf{H}}_{i} \mathbf{Q}_{i} \tilde{\mathbf{H}}_{i}^{H}\right)^{-2} \tilde{\mathbf{H}}_{i}+\lambda_{1} \boldsymbol{\Psi}_{i}+\lambda_{2} \mathbf{I}_{N_{s_{i}}}=0$.

By introducing an invertible matrix $\mathbf{K}_{i}$ with $\mathbf{K}_{i} \mathbf{K}_{i}^{H}=\lambda_{1} \boldsymbol{\Psi}_{i}+$ $\lambda_{2} \mathbf{I}_{N_{s_{i}}}$, (30) becomes

$$
\mathbf{K}_{i}^{-1} \tilde{\mathbf{H}}_{i}^{H}\left(\mathbf{D}_{i}+\tilde{\mathbf{H}}_{i} \mathbf{Q}_{i} \tilde{\mathbf{H}}_{i}^{H}\right)^{-2} \tilde{\mathbf{H}}_{i} \mathbf{K}_{i}^{-H}=\mathbf{I}_{N_{s_{i}}} .
$$

Obviously, (31) is valid if and only if

$$
\mathbf{K}_{i}^{-1} \tilde{\mathbf{H}}_{i}^{H}=\mathbf{P}_{i}\left(\mathbf{D}_{i}+\tilde{\mathbf{H}}_{i} \mathbf{Q}_{i} \tilde{\mathbf{H}}_{i}^{H}\right)
$$

where $\mathbf{P}_{i}$ is an $N_{s_{i}} \times N_{d}$ semi-unitary matrix with $\mathbf{P}_{i} \mathbf{P}_{i}^{H}=$ $\mathbf{I}_{N_{s_{i}}}$.

Let us introduce the following SVD and EVD

$$
\begin{aligned}
& \mathbf{K}_{i}^{-1} \tilde{\mathbf{H}}_{i}^{H}=\mathbf{U}_{i}\left[\begin{array}{cc}
\boldsymbol{\Sigma}_{i} & \mathbf{0}
\end{array}\right] \mathbf{V}_{i}^{H} \\
& \mathbf{D}_{i}+\tilde{\mathbf{H}}_{i} \mathbf{Q}_{i} \tilde{\mathbf{H}}_{i}^{H}=\left[\begin{array}{ll}
\mathbf{L}_{i, 1} & \mathbf{L}_{i, 2}
\end{array}\right] \operatorname{Bdiag}\left[\boldsymbol{\Delta}_{i, 1}, \boldsymbol{\Delta}_{i, 2}\right] \mathbf{L}_{i}^{H}(33)
\end{aligned}
$$

where the dimensions of $\mathbf{U}_{i}, \mathbf{V}_{i}, \mathbf{L}_{i}$ are $N_{s_{i}} \times N_{s_{i}}, N_{d} \times N_{d}$, and $N_{d} \times N_{d}$, respectively, $\mathbf{L}_{i, 1}$ and $\mathbf{L}_{i, 2}$ contain the leftmost $N_{s_{i}}$ columns and the rightmost $N_{d}-N_{s_{i}}$ columns of $\mathbf{L}_{i}$, respectively, and $\boldsymbol{\Sigma}_{i}, \boldsymbol{\Delta}_{i, 1}, \boldsymbol{\Delta}_{i, 2}$ are $N_{s_{i}} \times N_{s_{i}}, N_{s_{i}} \times N_{s_{i}}$, and $\left(N_{d}-N_{s_{i}}\right) \times\left(N_{d}-N_{s_{i}}\right)$ diagonal matrices, respectively. Substituting (33) back into (32), we have

$$
\mathbf{U}_{i}\left[\begin{array}{ll}
\boldsymbol{\Sigma}_{i} & \mathbf{0}
\end{array}\right] \mathbf{V}_{i}^{H}=\left[\begin{array}{ll}
\mathbf{P}_{i} \mathbf{L}_{i, 1} \boldsymbol{\Delta}_{i, 1} & \mathbf{P}_{i} \mathbf{L}_{i, 2} \boldsymbol{\Delta}_{i, 2}
\end{array}\right] \mathbf{L}_{i}^{H} .
$$

Equation (34) holds if and only if $\mathbf{P}_{i}=\mathbf{U}_{i} \mathbf{L}_{i, 1}^{H}, \boldsymbol{\Delta}_{i, 1}=\boldsymbol{\Sigma}_{i}$, and $\mathbf{L}_{i}=\mathbf{V}_{i}$. Thus, from (33) we have that

$$
\mathbf{D}_{i}+\tilde{\mathbf{H}}_{i} \mathbf{Q}_{i} \tilde{\mathbf{H}}_{i}^{H}=\mathbf{V}_{i} \mathbf{J}_{i} \mathbf{V}_{i}^{H}
$$

where $\mathbf{J}_{i} \triangleq \operatorname{Bdiag}\left[\boldsymbol{\Sigma}_{i}, \boldsymbol{\Delta}_{i, 2}\right]$. Let us introduce the SVD of $\tilde{\mathbf{H}}_{i}$ as

$$
\tilde{\mathbf{H}}_{i}=\left[\begin{array}{ll}
\mathbf{U}_{h_{i}, 1} & \mathbf{U}_{h_{i}, 2}
\end{array}\right]\left[\begin{array}{ll}
\boldsymbol{\Lambda}_{h_{i}, 1} & \mathbf{0}
\end{array}\right]^{T} \mathbf{V}_{h_{i}}^{H}
$$

where the dimensions of $\mathbf{U}_{h_{i}, 1}, \mathbf{U}_{h_{i}, 2}, \mathbf{V}_{h_{i}}$ are $N_{d} \times N_{s_{i}}$, $N_{d} \times\left(N_{d}-N_{s_{i}}\right)$, and $N_{s_{i}} \times N_{s_{i}}$, respectively, $\boldsymbol{\Lambda}_{h_{i}, 1}$ is an $N_{s_{i}} \times$ $N_{s_{i}}$ diagonal matrix. By substituting (36) back into (35) and solve (35) for $\mathbf{Q}_{i}$, we have $\mathbf{Q}_{i}=\mathbf{V}_{h_{i}} \boldsymbol{\Lambda}_{h_{i}, 1}^{-1} \mathbf{U}_{h_{i}, 1}^{H}\left(\mathbf{V}_{i} \mathbf{J}_{i} \mathbf{V}_{i}^{H}-\right.$ $\left.\mathbf{D}_{i}\right) \mathbf{U}_{h_{i}, 1} \boldsymbol{\Lambda}_{h_{i}, 1}^{-1} \mathbf{V}_{h_{i}}^{H}$. Finally, taking into account the constraint (29), we obtain (25).

\section{REFERENCES}

[1] B. Rankov and A. Wittneben, "On the capacity of relay-assisted wireless MIMO channels," in Proc. 5th IEEE Workshop Signal Process. Advances in Wireless Commun., Lisbon, Portugal, Jul. 2004, pp. 323-327.

[2] B. Wang, J. Zhang, and A. Høst-Madsen, "On the capacity of MIMO relay channels," IEEE Trans. Inf. Theory, vol. 51, pp. 29-43, Jan. 2005.

[3] X. Tang and Y. Hua, "Optimal design of non-regenerative MIMO wireless relays," IEEE Trans. Wireless Commun., vol. 6, pp. 1398-1407, Apr. 2007.

[4] O. Muñoz-Medina, J. Vidal, and A. Agustín, "Linear transceiver design in nonregenerative relays with channel state information," IEEE Trans. Signal Process., vol. 55, pp. 2593-2604, Jun. 2007.

[5] W. Guan and H. Luo, "Joint MMSE transceiver design in nonregenerative MIMO relay systems," IEEE Commun. Lett., vol. 12, pp. 517-519, Jul. 2008.

[6] A. S. Behbahani, R. Merched, and A. M. Eltawil, "Optimizations of a MIMO relay network," IEEE Trans. Signal Process., vol. 56, pp. 50625073, Oct. 2008.

[7] Y. Rong, X. Tang, and Y. Hua, "A unified framework for optimizing linear non-regenerative multicarrier MIMO relay communication systems," IEEE Trans. Signal Process., vol. 57, pp. 4837-4851, Dec. 2009.

[8] T. Tang, C.-B. Chae, R. W. Heath, Jr., and S. Cho, "On achievable sum rates of a multiuser MIMO relay channel," in Proc. IEEE ISIT, Seattle, USA, Jul. 2006, pp. 1026-1030.

[9] C.-B. Chae, T. Tang, R. W. Heath, Jr., and S. Cho, "MIMO relaying with linear processing for multiuser transmission in fixed relay networks," IEEE Trans. Signal Process., vol. 56, pp. 727-738, Feb. 2008.

[10] J. Yu, D. Liu, C. Yi, and G. Yue, "Relay-assisted MIMO multiuser precoding in fixed relay networks," in Proc. Int. Conf. Wireless Commun., Networking Mobile Comput., Shanghai, China, Sep. 2007, pp. 881-884.

[11] L. Weng and R. D. Murch, "Multi-user MIMO relay system with self-interference cancellation," in Proc. IEEE Wireless Commun. and Networking Conf., Kowloon, China, Mar. 2007, pp. 958-962.

[12] G. Li, Y. Wang, T. Wu, and J. Huang, "Joint linear filter design in multiuser non-regenerative MIMO-relay systems," in Proc. IEEE Int. Conf. Commun., Dresden, Germany, Jun. 2009.

[13] M. R. A. Khandaker and Y. Rong, "Performance measure of multiuser detection algorithms for MIMO relay network," in Proc. 10th Postgraduate Electrical Engineering \& Computing Symposium, ECU, WA, Australia, Oct. 2009.

[14] Y. Yu and Y. Hua, "Power allocation for a MIMO relay system with multiple-antenna users," IEEE Trans. Signal Process., vol. 58, pp. 28232835, May 2010.

[15] S. M. Kay, Fundamentals of Statistical Signal Processing: Estimation Theory. Englewood Cilffs, NJ: Prentice Hall, 1993.

[16] S. Boyd and L. Vandenberghe, Convex Optimization. Cambridge, U. K.: Cambridge University Press, 2004.

[17] M. Grant and S. Boyd, "Cvx: Matlab software for disciplined convex programming (web page and software)." http://cvxr.com/cvx, April, 2010. 